\title{
HISTOMORPHOLOGICAL CHARACTERISTICS OF ASCARIS LUMBRICOIDES AND TAENIARHYNCHUS SAGINATUS
}

\author{
Kristian Yeremiev', Tatyana Cvetkova ${ }^{2}$, Stoyan Pavlov ${ }^{3}$, Kalina Stoyanova ${ }^{2}$ \\ ${ }^{1}$ Student, Medical College, Medical University of Varna \\ ${ }^{2}$ Department of Infectious Diseases, Parasitology and Dermatovenerology, \\ Faculty of Medicine, Medical University of Varna \\ ${ }^{3}$ Department of Anatomy, Histology and Embryology, Faculty of Medicine, \\ Medical University of Varna
}

\begin{abstract}
INTRODUCTION: Ascaridosis and taeniarhynchosis are intestinal parasitic diseases, caused by the multicellular helminthes - Ascaris lumbricoides and Taeniarhynchus saginatus. A. lumbricoides is the largest nematode found in the human intestines with the female organism reaching lengths between 20 and 40 centimeters. T. saginatus is a hermaphroditic cestode reaching lengths from 6 up to 10 meters.

Aim: The aim of the current study was to prepare histological tissue sections of both intestinal parasites and to present their specific microscopic structure using different staining techniques.

MATERIALS AND METHODS: Two museum preparations of the adult worms, fixed in a $10 \%$ formalin solution were used. Histological tissue sections were prepared and stained with the hemalaun-eosin technique, Heidenhein's azan, van Gieson's trichrome and acid orcein after Taenzer-Unna stains.

RESULTS: The morphology and positioning of the internal organs of the intestinal parasites were clearly visualized by the hemalaun-eosin technique, while the additional stains allowed the differentiation of some specific morphological elements like the fine structure of the cuticle of $A$. lumbricoides, cestodal eggs' morphology, etc.

CONCLUSION: Although the routine diagnostic procedures for intestinal parasites are more efficient, the histological samples stained with different techniques can provide useful information in the ongoing education of the specialists in human and veterinarian parasitology and biology.
\end{abstract}

Keywords: Ascaris lumbricoides, Taeniarhynchus saginatus, histological sample preparation, hemalauneosin, van Gieson, azan

\author{
Address for correspondence: \\ Kristian Yeremiev \\ Department of Infectious diseases, Parasitology and \\ Dermatovenerology \\ Faculty of Medicine \\ Medical University of Varna \\ 55 Marin Drinov St \\ 9002 Varna, Bulgaria \\ e-mail:kristianyeremiev@gmail.com
}

Received: September 19, 2016

Accepted: November 8, 2017

\section{INTRODUCTION}

Ascaridosis and taeniarhynchosis are chronic parasitic infections (helminthiases). They may present with variety of symptoms in different patients depending on the worm burden, and the immunologic and nutritional status of the human host. Ascaridosis is caused by the roundworm Ascaris lumbricoides, a member of family Ascarididae, phylum Nematoda. It is dioecious, spindle-shaped, with creamywhite or yellowish color and is the largest nematode 
parasitizing in the human intestinal tract. The female organism reaches lengths from 20 up to 40 centimeters and has a conically pointed posterior end. The males reach size between 15 and 20 centimeters and have a ventrolaterally curved, hook-like posterior end (1-3). Taeniarhynchosis is caused by Taeniarhynchus saginatus (commonly known as beef tapeworm), taxonomically classified in the Taeniidae family, order Cyclophyllidea, class Cestoda, phylum Plathelminthes. Macroscopically, the strobila is segmented and composed of a scolex, colon and multiple hermaphroditic proglottids. The colon constantly generates new proglottids, replacing the ones that detach from the taenia body via apolysis and are shed with the stool. All proglottids are dorsoventrally flattened and bilaterally symmetrical, the proximal ones are squared while the distal ones are more elongated. The proximal proglottids possess both male and female rudimentary reproductive organs which develop fully in the intermediate segments. The main feature of the distal proglottids is the fully developed uterus packed with thousands of fertilized eggs, while the other organs have undergone atrophy. The high number of the uterine bilateral branches is a major species determinant, which allows its immediate differentiation from Taenia solium (pork tapeworm) (2-4).

\section{AIM}

The aim of the current study was to prepare histological tissue sections from the mature worms using different staining techniques to present their general anatomy, as well as the histological structure of the separate tissues and organs.

\section{MATERIALS AND METHODS}

For the histological preparations museum specimens of $A$. lumbricoides and T. saginatus were used. The helminths were evacuated from two patients after etiological treatment and the specimens were fixed and conserved via immersion in a $10 \%$ formol solution (about 3\% - 4\% formaldehyde). Six fragments of the mature A. lumbricoides were excised as well as several distal proglottids of T. saginatus. All samples were embedded in paraffin. The A. lumbricoides segments were sectioned transversely to the longitudinal axis while the T. saginatus proglottids were cut longitudinally. The calcium depos- its in the proglottids did not compromise the sectioning procedure, so no decalcification was necessary. The $4-6 \mu \mathrm{m}$ thick paraffin sections were mounted on microscopy slides and stained with four different staining protocols used routinely in histological and histo-pathological laboratories: (i) routine hemalaun-eosin staining (HE) with Gill's acidic hemalaun III and Eosin Y; (ii) a modified van Gieson's protocol (vG), using Gill's acidic hemalaun III, instead of Weigert's ferric hemalaun; (iii) Heidenhein's azan (aH) and (iv) Taenzer-Unna's acetic orcein (Or) $(5,6)$.

All the samples were scanned with an EC PlanNeofluar $40 x / 0.75$ objective on a motorized light microscope "Axio Imager Z2" (Carl Zeiss GmbH, Jena, Germany) and high definition $(0.16 \mu \mathrm{m} / \mathrm{px})$ digital images of the entire sections were prepared.

\section{RESULTS}

Ascaris lumbricoides: The cross-sections from A. lumbricoides revealed the ultrastructure of the parasite on two levels. The anterior sections were identified by the centered foregut (esophagus) (Fig. 1) and the intermediate sections confirmed that the worm was a mature female specimen (Fig. 2).

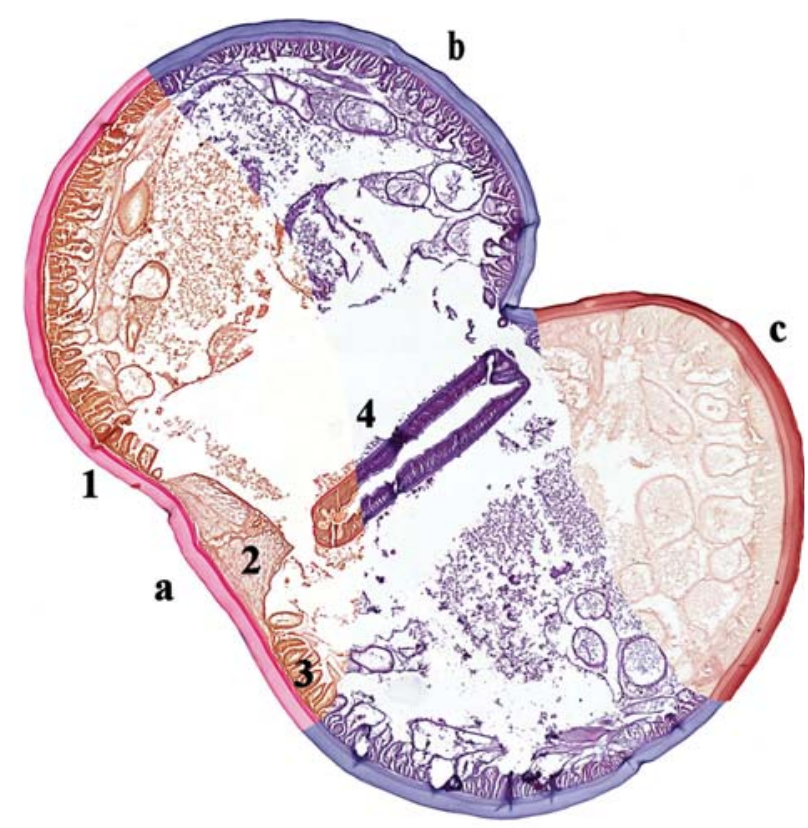

Fig. 1. Overview of cross-sectional histological organization of anterior end of A. lumbricoides. A synthetic image created by combining segments of the differently stained sections: $a$. van Gieson's trichrome $(v G), b$. hemalaun-eo$\sin (H E)$, c. acid orcein after Taenzer-Una (Or), 1-cuticle, 2-lateral chords, 3-myocytes, 4-esophagus. Obj. 40x/0.75 


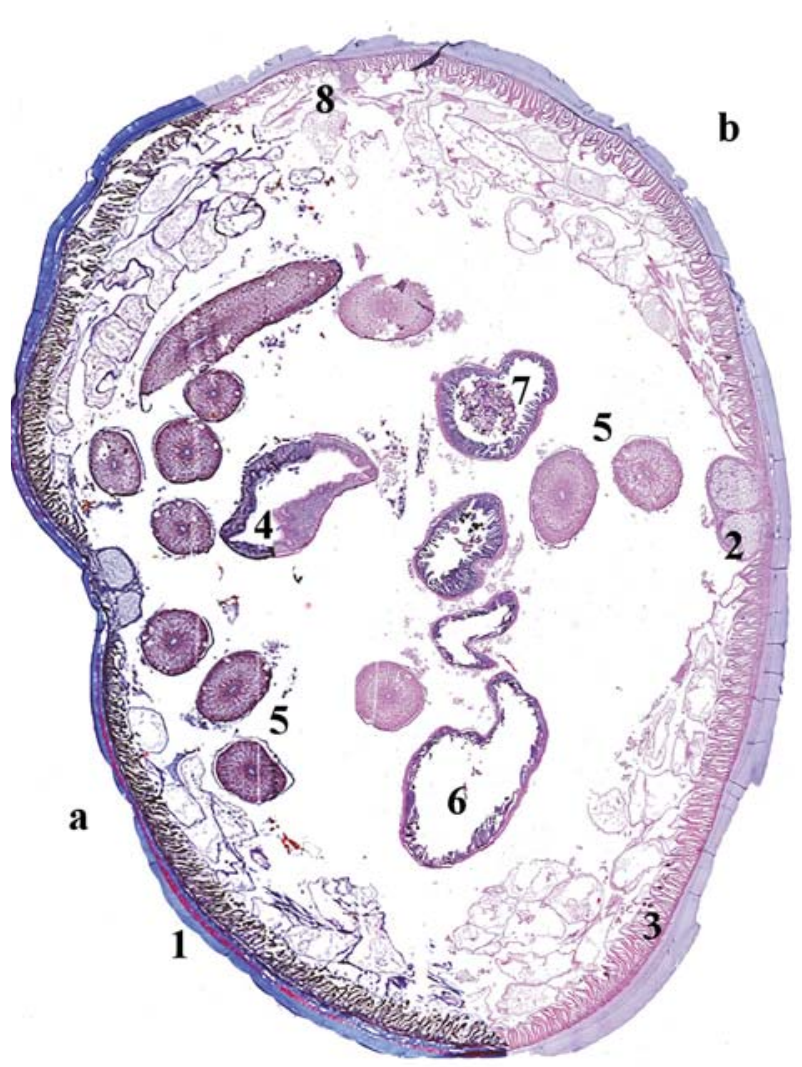

Fig. 2. Overview of cross-sectional histological organization of an intermediate section of A.lumbricoides. A synthetic image created by combining segments of the differently stained sections: $a$. Haidenhein's azan (aH), b. HE;1cuticle, 2-lateral chords, 3-myocytes, 4-intestine, 5-ovaries, 6-uterus, 7-ova, 8-ventral nerve. Obj. 40x/0.75

The fine structure of the cuticle (1, Fig. 1 and $\mathbf{1}$, Fig. 2) is clearly presented in all staining protocols. In the hemalaun-eosin stained samples it has a basophilic appearance (b, Fig. 1 and Fig. 2). All four sublayers of the cuticle are clearly visible with the top showing the most prominent basophily. The epicuticle's reaction is probably due to the high content of glycoproteins and sulfated proteoglycans (7). The high collagenous content of the cuticle is evident from its intense fuchsin positive staining in the sections processed with van Gieson's protocol (a, Fig. 1), and the intense aniline blue positive appearance in the azan-stained sections (a, Fig. 2). The cuticlins are the second major class of proteins in the nematode cuticle (8). They are highly cross-linked mainly via tyrosine and disulfide bonding $(7,8)$. The latter can explain the highly positive orcein staining of the cuticle (c, Fig. 1) $(9,10)$. The radial striations of the cortical zone and the four sheets of spiral fibers in the outer basal zone are most prominent in the orcein and azan stains. In the azanstained sections, the spiral fibers in the outer basal sublayer of the cuticle are highly azocarmine positive (1, Fig. 2). Subjacent to the cuticle is the dense, fleshy hypodermis (subcuticle). The lateral chords (2, Fig. 1 and 2, Fig. 2) are surrounded by the hypodermis and show a paler staining. The sections stained with van Gieson's trichrome revealed a clear contrast between the collagen-rich cuticle and the subjacent layers. The hypodermis overlays longitudinally and transversally oriented myocytes. The detailed view of the cross-sections reveals the U-shaped myocytes (3, Fig. 1 and Fig. 2) with visible striations and peripherally positioned nuclei $(11,12)$. The cuticle, hypodermis and muscle layer comprise the body cavity, which is filled with mesenchyme-derived tissue, which supports the internal organs. The anterior cross-sections revealed a flattened foregut (esophagus) (4, Fig. 1). In the intermediate sections we observed an asymmetrical, rounded intestine (4, Fig. 2) with multiple invaginations. The entire gastrointestinal tract is comprised of columnar epithelium with a visible apical brush border and a collagenous lamina basalis underlying the epithelial cells. The female reproductive system is presented by the elongated tubeshaped ovaries, oviducts and uterus. The ovaries (5, Fig. 2) are comprised of polygonal eosinophilic cells with clearly visible nuclei, which when observed under a higher magnification are seen to possess nucleoli. The posterior cross sections revealed an asymmetrical elongated uterus (6, Fig. 2) with a large lumen and smaller, rounded oviducts both possessing an inner lining of simple columnar epithelium underlined with a basal lamina. We observed multiple undeveloped, polymorphic ova in the uterine lumen (7, Fig. 2). The medial sections allowed observation of the ventral nerve of the parasite (8, Fig. 2). While the overall structure was relatively well preserved, there were signs of differently advanced autolysis in the sample due to the fixing of the whole specimen for museum preservation. Since the cuticle of the nematodes is highly impenetrable this probably slowed down the fixative's diffusion and allowed for post mortem autolysis to develop in the body cavity and the reproductive organs. For further use this can be easily countered via slow fixing at $4^{\circ} \mathrm{C}$. 
Taeniarhynchus saginatus: In this study, we prepared longitudinal tangential sections from several distal proglottids (Fig. 3). ate host (13). Besides hemalaun, azocarmine also revealed affinity towards the corpuscles, while the acetic orcein barely visualized them. The detailed imag-

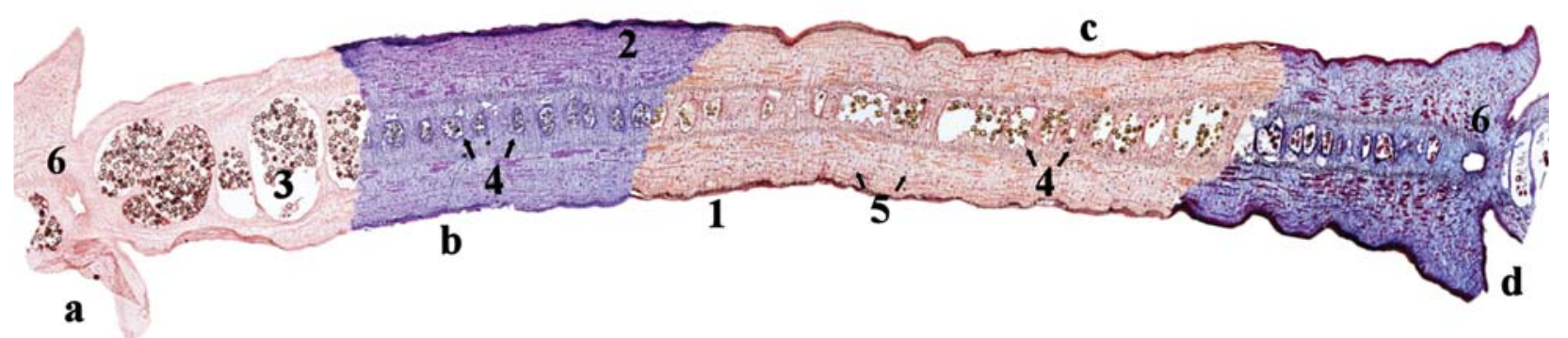

Fig. 3. Overview of histological organization of longitudinal sections of distal proglottids of T. saginatus. A synthetic image created by combining segments of the differently stained sections: a. Or, b. HE, c. vG, d. aH, 1-tegument, 2-myocytes, 3-uterus, 4-uterine branches, 5-calcareous corpuscles. Obj.40x/0.75

The thick eosinophilic syncytial outer layer of the tegument and the columnar epitheloid subtegumental cells are clearly discernable via the HE, vG and azan staining (1, Fig. 3). Underneath the tegument, the outer parenchymal layer is found, overlaying several muscular layers, comprised of transversally and longitudinally oriented myocytes (2, Fig. 3). These muscle layers determine the separate proglottids' active movements. Like all cestodes, T. saginatus does not possess a digestive system and acquires the necessary nutrients with the surface of the tegument. As aforementioned, the distal proglottids are sexually matured and as such, most of their internal organs are atrophied, leaving the most prominent structure - the gravid uterus (3, Fig. 3). Its large, bilaterally branched cavity is lined with simple columnar epithelium. The tangential sections show us an overview of the uterine morphology, while the longitudinal ones allow us to count the central branches (4, Fig. 3). These bilateral branches are a distinct structural characteristic and a major species determinant, allowing the differentiation of T. saginatus (with 1232 uterine branches) from T. sollium (between 8 and 12). The space between the separate organs is comprised of parenchyma, in which multiple small, circular, basophilic masses are observed. These masses are termed calcareous corpuscles and are comprised of concentric layers of calcium carbonate $\left(\mathrm{CaCO}_{3}\right)(\mathbf{5}$, Fig. 3). The calcareous corpuscles' purpose is still debated, but they are hypothesized to serve as calcium deposits, aiming to prolong the lifespan of the cysticerci, disseminated in the tissues of the intermedi- es were able to reveal on both posterior and distal end of the gravid proglottide, the formation of the region where the proglottid detachment, a process termed apolysis, will take place (6, Fig. 3).

All of the implemented staining techniques showed excellent results regarding the cestodal ova (Fig. 4).

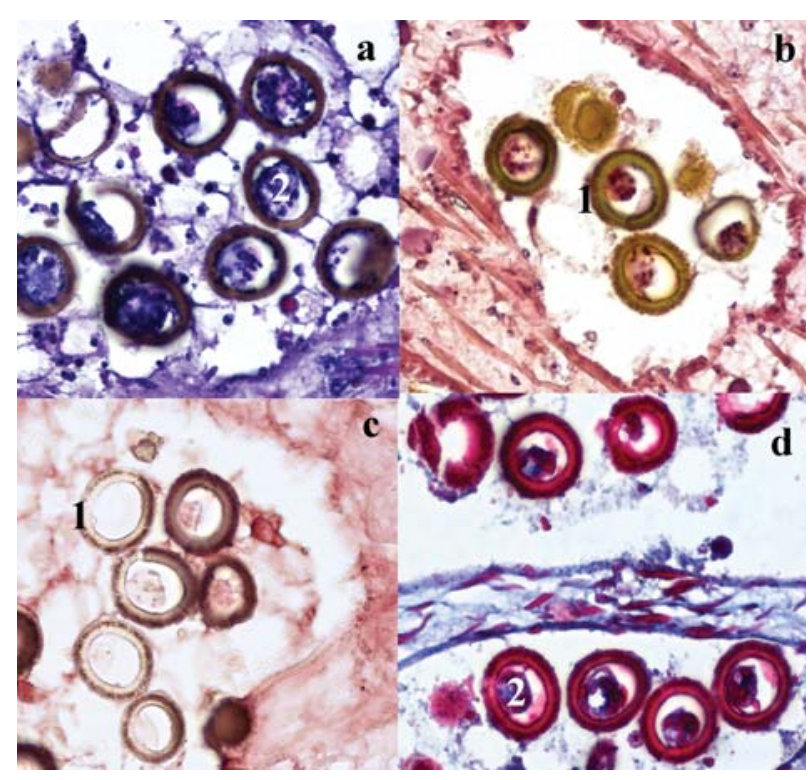

Fig. 4. Ova of T. saginatus in uterus. a. HE, b. vG, c. Or, d. aH, 1-shell, 2-oncospheres, Obj. 40x/0.75

The characteristic radially striated, double-layered shell (1, Fig. 4) which holds the oncosphere, is composed of keratin and stains very intensely with acetic orcein and azocarmine in the azan proto- 
col (d, Fig. 4). Regarding the oncospheres (2, Fig. 4), hemalaun-eosin, van Gieson's trichrome stain and Heidenhein's azan stain showed good results, while Taenzer-Unna's acetic orcein (c, Fig. 4) left the embryos unstained, giving the appearance of an empty shell. The specific structure of the cestodal eggs unfortunately cannot be used for the clinically important parasite genus differentiation $(4,14)$.

\section{CONCLUSION}

The museum formalin-preserved samples proved to be a useful source for the preparation of histological slides for the demonstration of the internal structure of both round- and tapeworms. The slow fixative diffusion and the consequent autolysis can be easily prevented by keeping the specimen at a $4^{\circ} \mathrm{C}$ temperature during the first few weeks of the specimen fixation. The histological samples and the unique detailed overview images obtained with the motorized Axio Imager Z2 microscope allowed a thorough observation on the parasites' internal morphology, giving a detailed overview of their anatomy, as well as some insights in the separate structures' cytological and histological characteristics. Although more efficient tests for intestinal parasites are implemented in the routine diagnostic practice, the histological examination can be helpful regarding the parasites' internal morphology especially when genus specific characteristics like the number of uterine branches are present. The derived images present a substantial enrichment of the educational image collection of Medical University-Varna and can be used in the ongoing education of parasitology residents, human and veterinarian medicine students and medical laboratory assistants. To our knowledge, this is the first documented experiment describing the application of Taenzer-Unna's acetic orcein stain on tissue sections from intestinal helminthes.

\section{Conflict of Interest}

The authors declare no conflict of interest

\section{REFERENCES}

1. Dold C, Holland CV. Ascaris and ascariasis. Microbes Infect Inst Pasteur. 2011;13(7):632-7.

2. Gillespie SH, Pearson RD. Principles and practice of clinical parasitology. Chichester, New York: Wiley; 2001.
3. Petrov P, Kurdova R, editors. Clinical parasitology and tropical medicine. 1st ed. Iztok-Zapad; 2016 (in Bulgarian).

4. Mayta H, Talley A, Gilman RH, Jimenez J, Verastegui M, Ruiz M, et al. Differentiating Taenia solium and Taenia saginata Infections by Simple Hematoxylin-Eosin Staining and PCR-Restriction Enzyme Analysis. J Clin Microbiol. 2000;38(1):133-7.

5. Welsch U, Mulisch M. Romeis Mikroskopische Technik. Spektrum Akademischer Verlag GmbH; 2010. 551 p.

6. Pavlov S, Goranova V. Histological technique. 1st ed. Steno; 2015 (in Bulgarian).

7. Page AP, Johnstone IL. The cuticle. WormBook [Internet]. 2007 [cited 2016 May 3]; Available from: http://www.wormbook.org/chapters/www_cuticle/ cuticle.html

8. Decraemer W, Karanastasi E, Brown D, Backeljau T. Review of the ultrastructure of the nematode body cuticle and its phylogenetic interpretation. Biol Rev Camb Philos Soc. 2003;78(3):465-510.

9. Deodhar KP, Tapp E, Scheuer PJ. Orcein staining of hepatitis B antigen in paraffin sections of liver biopsies. J Clin Pathol. 1975;28(1):66-70.

10. Woods GL, Walker DH. Detection of infection or infectious agents by use of cytologic and histologic stains. Clin Microbiol Rev. 1996;9(3):382-404.

11. Rosenbluth J. Ultrastructure of dyads in muscle fibers of Ascaris lumbricoides. J Cell Biol. 1969;42(3):817-25.

12. Rosenbluth J. Ultrastructure of somatic muscle cells in Ascaris lumbricoides. II. Intermuscular junctions, neuromuscular junctions, and glycogen stores. J Cell Biol. 1965;26(2):579-91.

13. von Brand T, Mercado TI, Nylen MU, Scott DB. Observations on function, composition, and structure of cestode calcareous corpuscles. Exp Parasitol. 1960;9(3):205-14.

14. Jimenez JA, Rodriguez S, Moyano LM, Castillo Y, García HH, Cysticercosis Working Group in Peru. Differentiating Taenia eggs found in human stools: does Ziehl-Neelsen staining help? Trop Med Int Health TM IH. 2010;15(9):1077-81. 